\title{
Melt conditioning by advanced shear technology (MCAST) for refining solidification microstructures
}

\author{
Z. Fan, M. Xia, H. Zhang, G. Liu, J. B. Patel, Z. Bian, I. Bayandorian, Y. Wang, \\ H. T. Li and G. M. Scamans
}

MCAST (melt conditioning by advanced shear technology) is a novel processing technology developed recently by BCAST at Brunel University for conditioning liquid metal prior to solidification processing. The MCAST process uses a twin screw mechanism to impose a high shear rate and a high intensity of turbulence to the liquid metal, so that the conditioned liquid metal has uniform temperature, uniform chemical composition and well-dispersed and completely wetted oxide particles with a fine size and a narrow size distribution. The microstructural refinement is achieved through an enhanced heterogeneous nucleation rate and an increased nuclei survival rate during the subsequent solidification processing. In this paper we present the MCAST process and its applications for microstructural refinement in both shape casting and continuous casting of light alloys.

Keywords: Melt conditioning, nucleation control, solidification, grain refinement

\section{Introduction}

Solidification of metals (casting) plays a crucial role in both scientific research and industrial development. Solidification developed as a technology during the Industrial Revolution and as a scientific subject from the early 1950's symbolised by the classical nucleation theory. ${ }^{1,2}$ Since then, tremendous progress has been made on the understanding of crystal growth during solidification and theoretical modelling of the solidification microstructure. ${ }^{3,4}$ However, the current shape cast components usually contain a coarse and non-uniform microstructure, various casting defects and severe chemical segregation, offering poor mechanical performance. For demanding applications, extensive thermo-mechanical processing must be applied to the continuously cast feedstock to achieve microstructural refinement and compositional uniformity. Such thermomechanical processing is usually low in materials yield, intensive in energy consumption, capital investment and manpower, and hence, inevitably high cost. A grand challenge is to develop solidification processing technologies which can ensure a fine and uniform as-cast microstructure free from macro-segregation and cast defects, so that the cast products can be either directly used in the as cast state, or only require minimal thermomechanical processing.

To address this grand challenge, over the recent years we have developed a novel solidification processing

BCAST, Brunel University, Uxbridge, Middlesex, UB8 3PH, UK

e-mail: zhongyun.fan@brunel.ac.uk technology named as MCAST (melt conditioning by advanced shear technology) for creation of fine and uniform solidification microstructures. ${ }^{5,6}$ In this paper we present the MCAST process and its application for microstructural refinement of light alloys.

\section{The MCAST process}

MCAST process is a novel technology for conditioning liquid metal prior to solidification processing. In the MCAST process, liquid metal is fed into a twin screw device, in which a pair of co-rotating and fully intermeshing screws are rotating inside a heated barrel with accurate temperature control. The liquid metal in the MCAST unit is subjected to intensive shearing under high shear rate and high intensity of turbulence. Consequently, the conditioned liquid metal has extremely uniform temperature, uniform composition and well dispersed inclusion particles. These individual particles will have a fine size and narrow size distribution, and more importantly, they are believed to be completely wetted by the liquid metal under the intensive forced convection (referred as forced wetting). The MCAST unit can be operated at temperatures either above the alloy liquidus to provided conditioned liquid metal, or below the alloy liquidus to provide semisolid slurry. In the latter case, the semisolid slurry contains a well controlled solid fraction of primary solid particles with a fine size and a spherical morphology. In addition, the MCAST process works either in a batch manner or in a continuous manner depending on the specific requirement of the subsequent casting processes. 

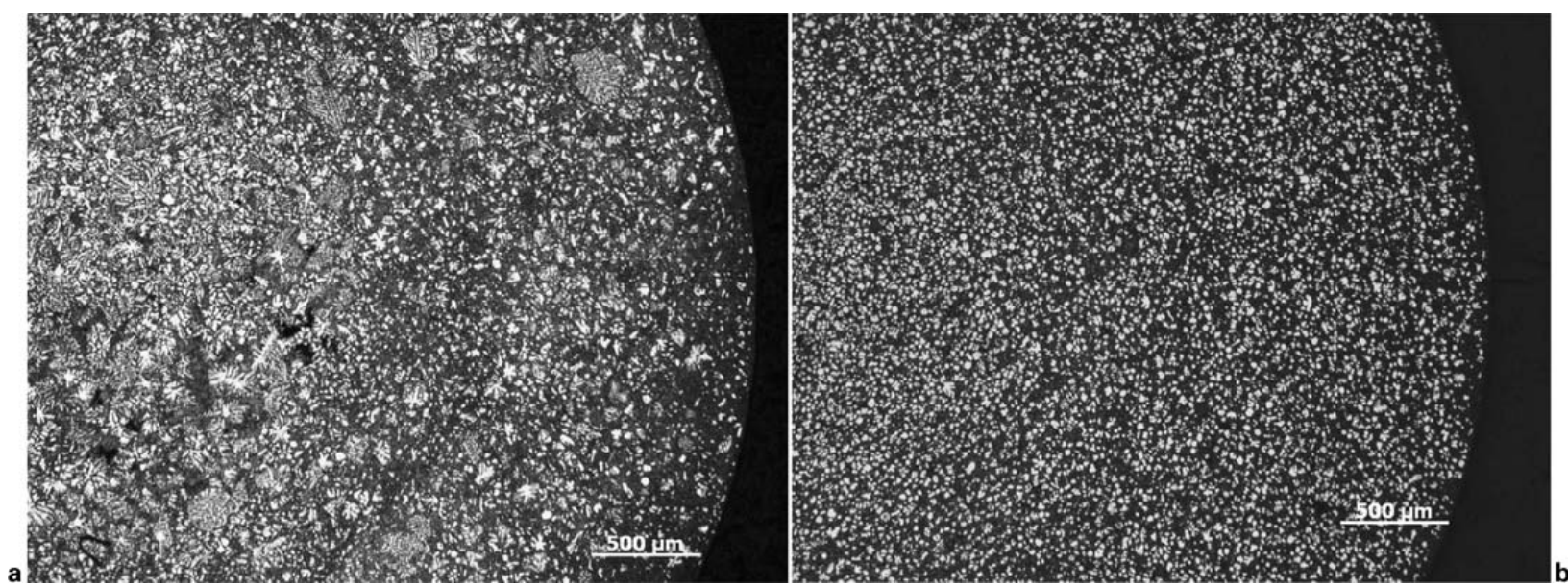

1 Microstructure of AZ91D alloy (cross section of a $\Phi 6 \mathrm{~mm}$ bar) produced by (a) the conventional HPDC and (b) the MCHPDC processes

Different cast products can be achieved by the attachment of the MCAST unit to different casting processes. The combination of the MCAST unit with the conventional HPDC process forms the MC-HPDC (melt conditioned high pressure die casting) for shape casting, or with the DC caster for the melt conditioned $\mathrm{DC}$ casting process (MC-DC process) to produce billet or slab, or a twin roll caster for the melt conditioned twin roll casting (MC-TRC) process for continuous strip production. Such technologies have been collectively called the MCAST technologies, which are applicable to both aluminium and magnesium alloys.

\section{Microstructures of MCAST light alloys}

AZ91D alloy (with a liquidus of $597^{\circ} \mathrm{C}$ ) melted at $700^{\circ} \mathrm{C}$ was fed into the MCAST unit and intensively sheared at $605^{\circ} \mathrm{C}$ and $800 \mathrm{rpm}$ for 45 seconds. The conditioned liquid metal was then fed into a standard cold chamber HPDC machine with a clamping force of 280 tonnes for producing standard dumbbell shaped tensile test samples with a gauge length of $60 \mathrm{~mm}$ and a diameter of
$6 \mathrm{~mm}$. The microstructure of the MC-HPDC sample is compared with that of a conventional HPDC sample in Fig. 1. Detailed microstructural characterisation revealed the following microstructural features of the MC-HPDC samples: ${ }^{7,8}$ (1) unlike the conventional HPDC sample (Fig. 1a) which contains substantial amount of porosity, the total porosity in the MCHPDC samples is $0 \cdot 2-0 \cdot 5 \%$, showing an order of magnitude reduction of porosity; (2) the globular primary particles with an average size of $20-40 \mu \mathrm{m}$ were uniformly distributed throughout the entire sample irrespective of the sample position and thickness (Fig. 1b); (3) the alloying elements are distributed uniformly throughout the MC-HPDC sample showing no macro-segregation; (4) the finally solidified eutectic phases were completely divorced in the MCAST AZ series Mg-alloys, and the $\beta$-phase had a fine size and a uniform distribution in the eutectic area.

The mechanical properties of AZ91D alloy processed by both the HPDC and the MC-HPDC processes at different casting temperatures are compared in Fig. 2. Fig. 2 shows that both the strength and ductility of the
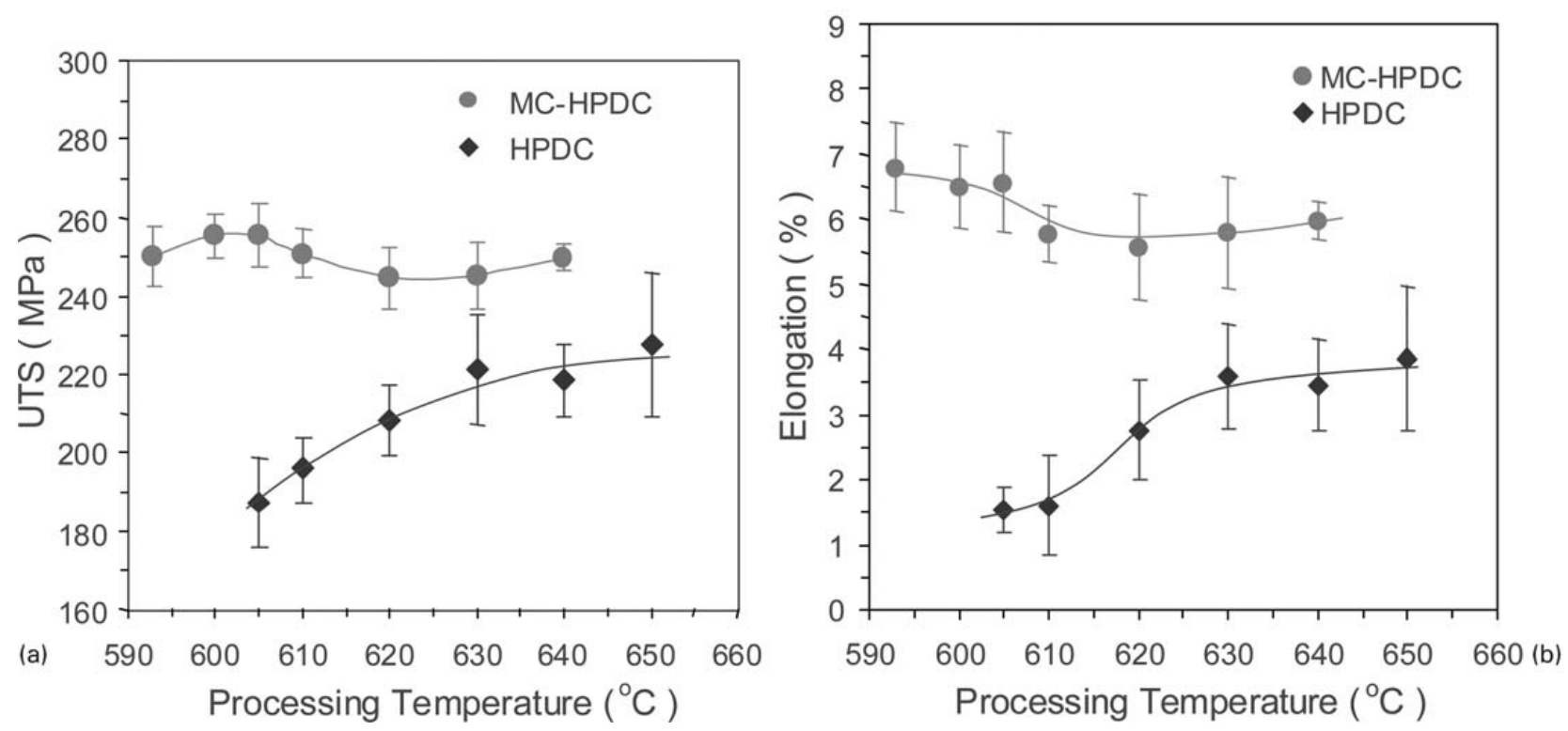

2 Mechanical properties of AZ91D alloy produced by the HPDC and the MC-HPDC processes as a function of the processing temperature 


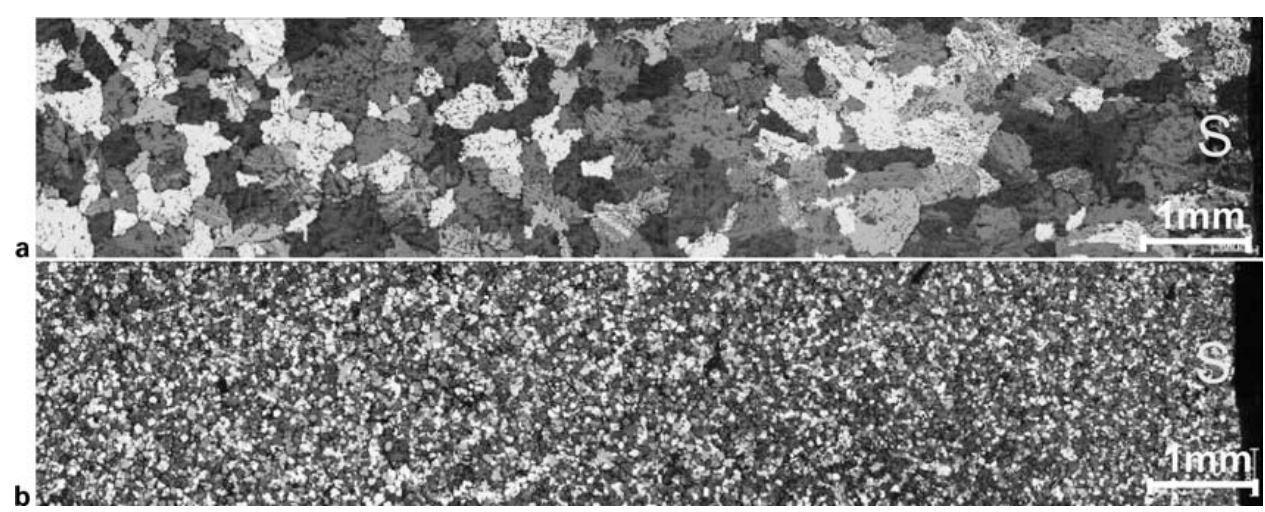

3 Microstructure of the modified AA5754 Al-alloy book mould sample with $40 \mathrm{~mm}$ thickness produced by (a) the conventional $\mathrm{DC}$ casting process and (b) by the MC-DC casting process. The letter ' $S$ ' marks the surface position of the samples

MC-HPDC samples are considerably higher than those produced by the conventional HPDC samples. In addition, both the strength and ductility of the HPDC samples decrease with decreasing casting temperature, showing a strong dependence on melt superheat. In contrast, the mechanical properties of the MC-HPDC samples are constantly high, showing little dependence on the superheat of the melt.

In order to simulate the MC-DC casting process, a modified AA5754 Al-alloy was sheared at $650^{\circ} \mathrm{C}$ and $500 \mathrm{rpm}$, and then cast into a book mould with a crosssection of $40 \times 200 \mathrm{~mm}$ and a pre-heating temperature of $350^{\circ} \mathrm{C}$. For comparison, the same alloy was cast under exactly the same conditions but without melt conditioning. For microstructural examination, transverse samples were cut from the centre of the book mould and then prepared using a standard metallographic technique before anodising to reveal the grain structure. The microstructure of the conditioned sample is compared in Fig. 3 to that of the conventional cast sample (without shearing). The conventionally cast samples have coarse columnar grains near the sample surface with an average grain size of $300 \mu \mathrm{m}$, severe macro-segregation and a substantial amount of hot tearing. In contrast, the conditioned samples have uniform fine and equiaxed grains with an average grain size of $70 \mu \mathrm{m}$ over the entire cross section, indicating that the intensive melt shearing refines the cast microstructure through enhanced heterogeneous nucleation. In addition, the conditioned samples have significantly reduced macrosegregation and hot tearing.

Fig. 4 compares the microstructures of the AZ91D alloy strip of $4 \mathrm{~mm}$ thickness processed by both conventional TRC and the MC-TRC processes. Without shearing, the TRC strip has a typical columnar grain structure with a grain size up to millimetre level (Figs. 4a and 4b). The columnar grain growth and the hot rolling process at the end of the TRC process lead to severe chemical segregation at the centre of the strip (called centre-line segregation) and other cast defects, such as hot cracks and surface bleeds (Fig. 4b). However, intensive melt shearing by the twin screw mechanism can eliminate the columnar growth giving rise to a fully equiaxed grain structure throughout the entire thickness (Figs. 4c and 4d). In addition, the MC-TRC sample shows no centre-line segregation and considerably reduced other casting defects.
Fig. 5 compares the microstructural variations in the as-cast AZ91D alloy strip produced by both the TRC and the MC-TRC processes. The TRC samples show a considerable variation of grain size between 200 and $600 \mu \mathrm{m}$ through the strip thickness. The relatively finer grain structure at the surface is due to the higher cooling rate, while the relatively finer grain size at the centre of the strip is a direct result of the increased solute contents at the centre of the strip, which provides a greater growth restriction. Intensive melt shearing can significantly refine the as-cast microstructure. The MC-TRC samples show a consistently fine microstructure with an average grain size of $60 \mu \mathrm{m}$ throughout the entire strip thickness.

\section{Discussion}

Experimental results presented in the previous section have demonstrated clearly that intensive melt shearing by the BCAST unit through a twin screw mechanism can achieve substantial microstructural refinement. The microstructural refinement of light alloys by the MCAST process can be understood from two different levels. At the macroscopic level, the fluid flow inside the MCAST unit is characterised by high shear rate and high intensity of turbulence. This intensive melt shearing ensures that both the temperature and chemical composition fields of the melt are extremely uniform throughout the entire volume of the melt. Under such conditions, heterogeneous nucleation will take place throughout the whole volume of the liquid metal, and all the nuclei created will have a chance to survive and eventually contribute to the microstructural refinement. ${ }^{6}$ This is in contrast to the conventional casting processes, where the majority of the nuclei created in the first place may remelt or dissolve once they are transferred to regions with temperatures higher than the alloy liquidus. In addition, at the microscopic level, intensive melt shearing will disperse the oxide films and clusters usually present in the alloy melt to individual oxide particles with a fine size and a narrow size distribution. More importantly, such dispersed oxide particles are completely wetted by the liquid metal under intensive forced convection. During the subsequent solidification processing, these oxide particle may either act directly as potent sites for heterogeneous nucleation of the primary phase, or act as potent nucleation sites for intermetallic particles, which then nucleate the primary phase through a multi-step nucleation mechanism, depending on the 


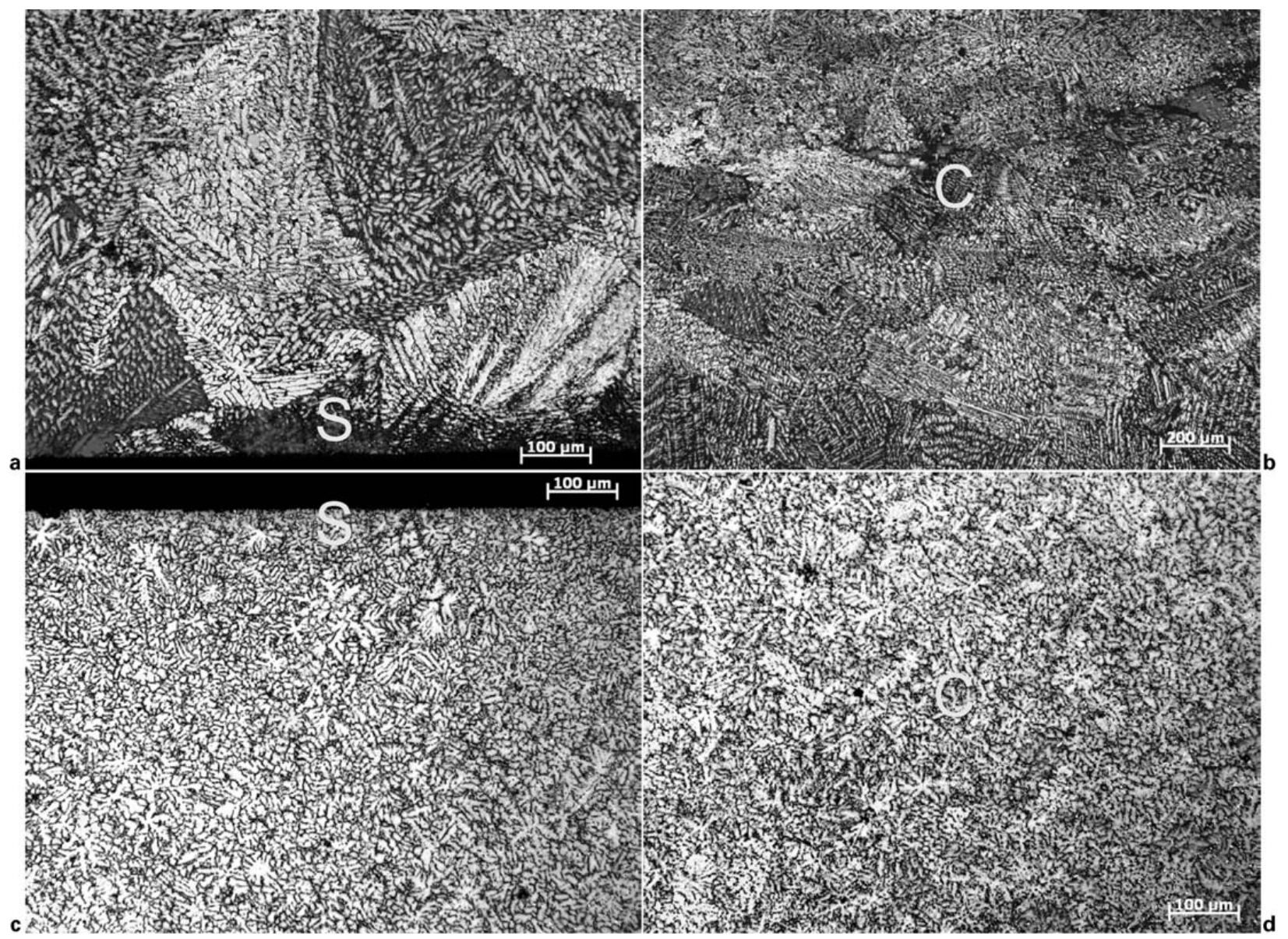

4 Microstructure of AZ91D alloy strip (4 mm thickness) produced by the conventional TRC process (a and b) and the MC-TRC process (c and d). The Letters ' $S$ ' and ' $C$ ' denote the surface and the centre of the strip, respectively. For the MC-TRC strip, the alloy melt was intensively sheared at $610^{\circ} \mathrm{C}$ and $500 \mathrm{rpm}$ prior to twin roll casting. Note that there are no columnar grains and central-line segregation in the MC-TRC strip.

nature of the oxide and the actual alloy compositions. This is discussed in more details in our paper on enhanced heterogeneous nucleation by intensive melt shearing in this volume. ${ }^{9}$

Microstructural refinement is achieved by intensive melt shearing provided by the MCAST process without any involvement of a deliberate chemical addition. This

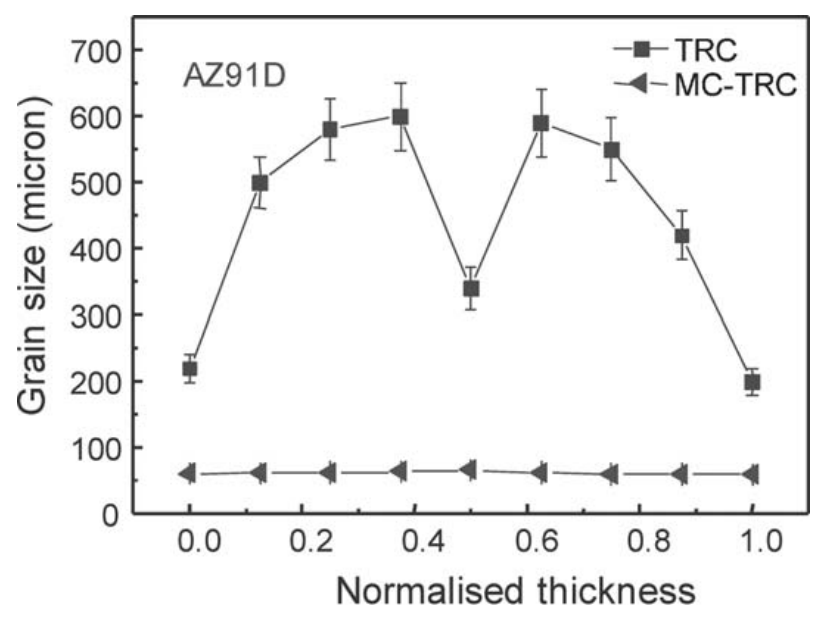

5 Through-thickness microstructural variation of AZ91D alloy strip ( $4 \mathrm{~mm}$ thickness) produced by the conventional TRC and the MC-TRC processes. For the MCTRC strip, the alloy melt was intensively sheared at $610^{\circ} \mathrm{C}$ and $500 \mathrm{rpm}$ prior to twin roll casting. can be called physical grain refinement in contrast to the chemical grain refining achieved by addition of grain refiners, such as Al-Ti-B master alloy. The advantages of the physical grain refining approach over the conventional chemical grain refining approach include:

- It is applicable to both cast and wrought alloys without any limitation to alloy composition and without any poisoning effect by alloying elements.

- It turns the usually harmful oxide inclusions into useful nucleation sites for grain refinement;

- It has no detrimental effect on down stream processing;

- It increases the tolerance to the impurity elements (e.g., iron), so more scrap metal can be directly recycled in-house without gong through chemical purification. ${ }^{10,11}$

- It does not contaminate the alloy and therefore does not cause any concern for further recycling.

\section{Summary}

A novel processing technology, the MCAST process, has been developed for conditioning liquid metal through intensive melt shearing provided by a twin screw mechanism. It has been demonstrated that the MCAST process can provide cast products with fine and uniform microstructure, uniform chemical composition and much reduced or eliminated cast defects. The microstructural refinement can be attributed to increased nuclei survival rate under extremely uniform

International Journal of Cast Metals Research 2009 VOL 22 NO 1-4 
temperature and composition fields at the macroscopic level and the enhanced heterogeneous nucleation rate by the well dispersed fine oxide particles with a narrow size distribution achieved through intensive melt shearing at the microscopic level. This microstructural refinement through intensive melt shearing has been called physical grain refining, in contrast to the conventional chemical grain refining by addition of grain refiners. Compared with chemical grain refinement, physical grain refining can be more effective, universally applicable with less harmful effect on down stream processing such as thermal mechanical processing and recycling.

\section{Acknowledgements}

The authors wish to thank MEL (Manchester, UK) for providing the AZ91D alloy ingots and Novelis
(Kingston, Canada) for providing the base AA5754 alloy billets. Financial support from both EPSRC (UK) and TSB (UK) is gratefully acknowledged.

\section{References}

1. D. Turnbull: J. Chem. Phys., 1952, 20, 411.

2. D. Turnbull: Acta Metall., 1953, 1, 8.

3. W. Kurz and D. J. Fisher: 'Fundamentals of Solidification', 3rd edn, Trans Tech. Publications, 1992, Switzerland.

4. A. L. Greer, A. M. Bunn, A. Tronche, P. V. Evans and D. J. Bristow: Acta Mater., 2000, 48, 2823.

5. Z. Fan, X. Fang and S. Ji: Mater. Sci. Eng. A, 2005, 412, 298.

6. Z. Fan and G. Liu: Acta Mater., 2005, 53, 4345.

7. Z. Fan, G. Liu and Y. Wang: J. Mater. Sci., 2006, 41, 3631

8. Y. Wang, G. Liu and Z. Fan: Acta Mater., 2006, 54, 689.

9. Y. Wang, Z. Fan et al: in this proceeding, 2008

10. G. Liu, Y. Wang, and Z. Fan: Mat. Sci. Eng. A, 2008, 472, 251

11. X. Fang, G. Shao, Y. Q. Liu and Z. Fan: Mater. Sci. Eng., 2007, 445-446, 65 . 


\section{Authors Queries}

Journal: International Journal of Cast Metals Research

Paper: Fan

Title: Melt conditioning by advanced shear technology (MCAST) for refining solidification microstructures

Dear Author

During the preparation of your manuscript for publication, the questions listed below have arisen. Please attend to these matters and return this form with your proof. Many thanks for your assistance

\begin{tabular}{|l|l|l|}
\hline $\begin{array}{l}\text { Query } \\
\text { Reference }\end{array}$ & Query & Remarks \\
\hline 1 & Please confirm the short title. & \\
\hline
\end{tabular}

